\title{
QUEEN'S
UNIVERSITY
BELFAST
}

\section{Mind the gap: Gender differences in walkability, transportation and physical activity in urban India}

Adlakha, D., \& Parra, D. (2020). Mind the gap: Gender differences in walkability, transportation and physical activity in urban India . Journal of Transport and Health. https://doi.org/10.1016/j.jth.2020.100875

Published in:

Journal of Transport and Health

Document Version:

Peer reviewed version

Queen's University Belfast - Research Portal:

Link to publication record in Queen's University Belfast Research Portal

\section{Publisher rights}

Copyright 2020 Elsevier.

This manuscript is distributed under a Creative Commons Attribution-NonCommercial-NoDerivs License

(https://creativecommons.org/licenses/by-nc-nd/4.0/), which permits distribution and reproduction for non-commercial purposes, provided the author and source are cited.

\section{General rights}

Copyright for the publications made accessible via the Queen's University Belfast Research Portal is retained by the author(s) and / or other copyright owners and it is a condition of accessing these publications that users recognise and abide by the legal requirements associated with these rights.

Take down policy

The Research Portal is Queen's institutional repository that provides access to Queen's research output. Every effort has been made to ensure that content in the Research Portal does not infringe any person's rights, or applicable UK laws. If you discover content in the Research Portal that you believe breaches copyright or violates any law, please contact openaccess@qub.ac.uk. 


\section{Abstract}

\section{Introduction}

Insufficient physical activity (PA) is a key contributor for premature mortality in low- and middleincome countries (LMICs). Despite the well-documented health benefits of PA, few women achieve the recommended levels of PA. The global average of inactivity among women is higher at $31.7 \%$ compared to $23.4 \%$ for inactive men. The gender gap in PA participation is wider in India where estimates indicate that $44 \%$ women are insufficiently active, compared to $25 \%$ men.

\section{Methods}

11 This cross-sectional study was conducted in the city of Chennai, India. Participants (N=370) were recruited from 155 wards stratified by neighborhood walkability and socio-economic status. The adapted Neighborhood Environment Walkability Scale for India (NEWS-India) was used to assess built environment perceptions on density, land-use mix, street infrastructure, aesthetics, traffic and crime safety. Travel and leisure PA were recorded using the International Physical Activity Questionnaire-Long Form (IPAQ-LF). Study protocols were based on recommendations of the International Physical activity and the Environment Network (IPEN; www.ipenproject.org) and previously used in studies in Brazil, Colombia, and Nigeria.

\section{Results}

21 A greater number of women reported achieving weekly PA levels from travel-related activities $(n=54,16.0 \%)$ compared to men $(n=33,9.8 \%)$. In contrast, a higher percentage of men $(n=75$, $22.3 \%)$ met weekly PA recommendations during leisure-time compared to women $(n=54$, 16.1\%). Street connectivity $(\mathrm{aOR}=3.2, \mathrm{Cl}=1.3,8.0)$ and land-use mix access $(\mathrm{aOR}=3.7, \mathrm{Cl}=1.4$, 8.2) significantly increased odds of travel PA among women.

\section{Conclusions}

28 Low engagement in leisure PA among women can be linked to prevailing socio-cultural norms that constrain women's mobility and hinder their participation in PA. Aspects of gender equity in 
1 transportation planning are closely linked with citywide indicators of liveability, sustainability and

2 resilience in LMICs like India. Public health practitioners must address gender-specific barriers

3 to increase PA among women through context-specific group exercise programs, peer support

4 and guidance that emphasize the importance of PA.

5

6 Keywords

7 non-communicable diseases; low- and middle-income countries; physical activity; built

8 environment; neighborhood walkability; India 


\section{Introduction}

2 Physical inactivity is a primary modifiable risk factor for non-communicable diseases (NCDs) such as heart disease, diabetes, obesity, stroke, hypertension, and some cancers. ${ }^{1}$ A quarter of adults are inactive across the globe, costing billions of dollars in healthcare expenditure and productivity losses. ${ }^{2}$ This epidemic is magnified in low- and middle-income countries (LMICs) that are currently experiencing the highest population growth rates. ${ }^{3}$ It is estimated that NCDs account for $80 \%$ of the global burden of disease in LMICs and insufficient physical activity (PA) is a key contributor for premature mortality in these populations. ${ }^{4}$ In India, an LMIC with a population of 1.4 billion people, less than $54.4 \%$ of the population meets the recommended PA guidelines and incidence rates for diabetes, heart disease and cancer are on

11 a steep rise..$^{5-7}$ NCDs currently account for $53 \%$ of the disease burden and $44 \%$ of disability12 adjusted life years (DALYs) lost in India. ${ }^{8}$ Despite the well-documented health benefits of PA,

13 few women achieve the recommended levels of PA. ${ }^{9}$ The global average of inactivity among 14 women is much higher at $31.7 \%$ compared to $23.4 \%$ for inactive men. ${ }^{9}$ The gender gap in PA participation is wider in India where estimates indicate that $44 \%$ of Indian women are insufficiently active, compared to $25 \%$ men. $^{10}$

Scientific guidelines and calls to action issued by various agencies such as the US Department of Health and Human Services, UK Department of Health, and the World Health Organization's Global NCD Action Plan recommend that 150 minutes of moderate intensity PA or 75 minutes of vigorous PA per week are necessary to achieve health benefits. ${ }^{11-13}$ Studies from developed nations have consistently shown that PA is affected by the interaction of individual, social, environmental and policy level factors. ${ }^{8}$ However, recommendations from these studies may not be applicable to LMICs like India with varying cultural contexts, social mores, gendered norms and distinct patterns of urban development and land-use. India's socio-cultural setting is similar to several countries in south and south-east Asia, yet Indian women face greater restrictions and threats to personal safety compared to their counterparts in Malaysia, Indonesia, 
1 Research has established that PA patterns and predictors vary by gender, yet research on the

2 barriers and supports of PA for women is limited. Compared to men, women are more likely to

3 report barriers to PA such as lack of time, financial constraints, body image negativity, and

4 socio-cultural norms, neighborhood disadvantage, risks to safety, low walkability and poor

5 quality of built environment (BE). ${ }^{15}$ Women's participation in outdoor PA is restricted by

6 gendered social norms in several communities and religious groups across India. Unsafe

7 neighborhood conditions, limited access to parks, playgrounds and open spaces, lack of time,

8 unsupportive pedestrian infrastructure, and cultural taboos are commonly cited constraints for

9 women's engagement in PA. ${ }^{8} 16$ Traditional socio-cultural norms have established predefined

10 responsibilities for women where they often play the lead role as caregivers or homemakers, in

11 addition to paid work outside the home, consequently impinging on their leisure time. ${ }^{17}$ Recent

12 reports have highlighted women's concerns of personal safety from gender violence and

13 harassment as limitations toward their use of public spaces such as parks, playgrounds and

14 streets, restricting their independence and participation in community events and socio-cultural

15 activities. $^{18}$

Research has established that transportation is a key factor that allows women to participate in the workforce and access social opportunities. ${ }^{19}$ In India, women and girls constitute $50 \%$ of the urban population and over $84 \%$ of trips by women are using public and non-motorized modes of transport. ${ }^{20}$ Women are likely to bear disproportionate impacts from poor BE infrastructure and public transport compared to men. While leisure PA such as going to a gym may be an additional burden, transport is a necessity of everyday life for women in India. Encouraging active transportation (walking and cycling) for utilitarian travel may be a feasible approach to increasing levels of PA in Indian women. ${ }^{19}$

A plethora of research has demonstrated that BE interventions aimed at increasing the amount of PA within a population are likely to have a positive impact on health and address the NCD epidemic. ${ }^{21}$ Global calls to action have exhorted LMICs to address the gender imbalance emerging from current patterns and trends in mobility, transport and urban health. ${ }^{22}$ In India, 
1 there has been an upsurge in research and policy advocacy to incorporate gender and cultural

2 perspectives into public health, education and the agricultural sectors, however few attempts

3 have focused attention in the transport sector in urban areas. Urban planning and public

4 transport investments in India have not considered the interrelationships between gender,

5 transport and health inequities. To date, limited research has examined gender-specific

6 differences in walkability, transportation and PA in urban India and evidence on PA practices

7 among women in is scarce. Studies conducted in a few states across India point to insufficient

8 PA levels in the population, however, gender-specific differences have not been fully

9 elucidated. ${ }^{8}$ This paper probes gender differences in PA in two key life areas or domains-

10 leisure and transport-and correlates of the BE including density, diversity of land-uses,

11 aesthetics, street design (e.g. pedestrian and bicycling infrastructure), traffic safety, and crime.

12 We draw attention to issues confronting women's mobility and transport and the barriers they

13 encounter for PA engagement in a metropolitan setting in India. This study fills a key gap in the

14 knowledge on gendered mobilities in urban India and makes a case for gender-inclusive

15 policies for urban transport across LMICs.

\section{Methods}

18 Study protocols, methods and measures were adapted from the International Physical activity

19 and the Environment Network (IPEN; www.ipenproject.org) recommendations, a global

20 organization that has developed standardized procedures for worldwide research on BE

21 correlates of PA. ${ }^{23}$ The Institutional Review Board at [university affiliation removed for peer

22 review] approved all study procedures.

\subsection{Study Site}

25 This study was conducted in the Chennai metropolitan area (164.48 sq. miles), capital of Tamil

26 Nadu state in south India, with a current population of 9.1 million. ${ }^{20}$ For purposes of

27 administration and governance, the metropolitan area of Chennai city is divided into 10 zones encompassing a total of 155 smaller electoral subdivisions also referred to as wards. The city of 
1 Chennai recorded a population growth of $6.98 \%$ in the previous Census period, making it the

24 th most populous metropolitan area in India and the $30^{\text {th }}$ most populous city in the world. ${ }^{20}$

\subsection{Sampling and Participant Recruitment}

5 A cross-sectional study design with a stratified two-stage sampling approach was used in this

6 study. In the first stage, administrative units or wards were stratified to maximize variance in

7 neighborhood walkability and socio-economic status (SES). Wards were ranked by high and

8 low walkability and SES to account for variability and maximize the variance between

9 neighborhoods. These wards were then crossed to produce a $2 \times 2$ matrix of four quadrants representing the following criteria: high-walkable/high-SES, high-walkable/low-SES, lowwalkable/high-SES, and low-walkable/low-SES. This method of stratification at the ward level has been consistently used in similar studies in order to maximize the contrast between areas with different walkability characteristics while taking SES into account. ${ }^{24}$ It ensured that groups with insufficient or inadequate representation were proportionately selected. ${ }^{25}$ In the second stage, efforts were made to recruit a diverse group of participants from wards distributed across the metropolitan area of Chennai city that matched the walkability and SES criterion. Protocols of sampling, stratification of wards, and participant recruitment have been published elsewhere and summarized below. ${ }^{26}$

Within each ward, study participants were recruited using a purposive sampling approach. The principal investigator identified a group of residents and key informants. Local residents included representatives from organized community groups such as neighborhood associations or neighborhood-watch groups, resident welfare groups and apartment owner association. Key informants included representatives from local government departments (e.g., Chennai Metropolitan Development Authority, Chennai Corporation, Transport Department), think tanks and non-profit organizations (e.g., Institute for Transportation and Development Policy, Chennai City Connect). This group of residents and key informants supported with the recruitment process by identifying other eligible participants through their professional and community networks. 
1 Inclusion and exclusion criteria for participant recruitment were outlined in consultation with

2 researchers who have conducted similar IPEN studies in Africa, Brazil, Colombia, Mexico and

3 China. ${ }^{25,27,28}$ Participants that met the following eligibility criteria were recruited for the study: (i)

4 must be currently residing in the metropolitan area of Chennai city; (ii) has been resident in

5 Chennai for at least 6 months prior to the survey date; (iii) current age between 18-65 years; (iv)

6 fluent in English or Tamil (official languages of the study site); (v) no signs of physical or

7 cognitive impairment; (vi) able to walk independently. Recruitment was limited to one participant

8 per household to ensure data measurements are independent.

10 A moderate-to-large effect size (effect size statistic $[\mathrm{d}]=0.75$ ) was used to calculate the sample 11 size. This estimate was in adherence with IPEN studies in similar LMIC contexts. ${ }^{25}$ Sample size 12 calculations estimated that 73 participants from each of the four stratified ward quadrants were 13 required to detect the specified effect size with greater than $80 \%$ power. Recruitment was 14 conducted until at least 75 eligible participants from each quadrant had been surveyed.

\subsection{Measures}

2.3.1. Built Environment Characteristics

18 The adapted Neighborhood Environment Walkability Scale for India (NEWS-India) was used to assess BE characteristics. ${ }^{16}$ Subscales of NEWS-India measure the following perceived BE characteristics: a) residential density (7 items), b) land-use mix diversity (43 items), c) land-use mix access (7 items), d) street connectivity (5 items), e) infrastructure for walking and bicycling (13 items), f) aesthetics (6 items), g) traffic safety (6 items), and h) safety from crime (4 items). Psychometric properties of NEWS-India subscales have been previously established with intraclass correlation coefficient scores higher than 0.75 , indicating excellent reliability. ${ }^{16}$

\subsubsection{Physical Activity Domains}

PA levels were measured using the travel and leisure modules of the International Physical Activity Questionnaire-Long Form (IPAQ-LF), a self-report measure. ${ }^{29}$ These modules of IPAQ- 
1 intensity PA during leisure and travel activities. The IPAQ-LF has been tested internationally for

2 reliability (Spearman's rho $\sim 0.8$ ) and criterion validity (median rho $\sim 0.3$ ) with satisfactory

3 results. $^{30}$

\subsubsection{Demographic Variables}

6 Validated scales for Indian contexts were used to capture participants' responses on age,

7 gender, marital status, education, employment, SES and religious affiliation.

\subsection{Data Analysis}

10 The IPEN study protocol was used for scoring NEWS-India survey items and subscales. All

11 NEWS-India items used a four-point Likert-type scale response ranging from 1 (strongly agree)

12 to 4 (strongly disagree). All items were positively scored (higher scores indicated a walkable

13 neighborhood) and response categories combined into "agree" (strongly agree, agree) and

14 "disagree" (disagree, strongly disagree). Data analysis was conducted using the Statistical

Package for the Social Sciences (SPSS) version $25^{31}$

We used logistic regression models to identify BE factors associated with meeting domainspecific PA levels ( $\geq 150$ minutes/week) during travel and leisure. Separate models were created for men, women and the pooled sample. Models were adjusted for age and SES in order to control for the confounding effects of these variables. ${ }^{25,28,32}$

\section{Results}

23 A total of 370 adults participated in this study. Comparisons of participant demographics with census data showed the study sample was older (median age $=35$ years vs. census median age $=28.1$ years $)$, had greater number of women $(54.2 \%$ vs $50.3 \%)$ and lower literacy rate $(87.0 \%$ vs. $90.2 \%)$ than residents of the wards in which participants lived. ${ }^{20}$ The majority of participants in this sample had a graduate or professional degree $(50.4 \%, n=183)$, were employed $(62.5 \%, n=222)$ and married $(61.5 \%, n=224)$. Table 1 highlights the demographic characteristics of the participants. 


\subsection{Built Environment Perceptions and Commute Characteristics}

2 Perceptions of the BE and commute characteristics stratified by gender are presented in Table

32 2. In this study, $40.8 \%$ of women reported lack of infrastructure for walking and cycling and

$433.9 \%$ reported feeling unsafe at night due to fear of crime. Compared to men, a higher

5 percentage of women $(32.9 \%, n=120)$ reported feeling threatened by crime rates in their

6 neighborhood. Safety from traffic was also a key factor affecting women's perceptions of the

7 BE. Compared to men, a higher proportion of women reported difficult walking conditions due to

8 the volume of traffic $(33.8 \%, n=123)$ and exhaust fumes from vehicular traffic $(37.9 \%, n=138)$.

9 Levels of walking/ cycling and active commuting were higher among women, whereas men 10 reported greater use of private transport $(32.8 \%, n=77)$.

\subsection{Meeting Physical Activity Targets}

13 Table 3 highlights the descriptive statistics of men and women that met the WHO

14 recommended PA targets. A greater number of women reported meeting WHO recommended levels of weekly PA from travel-related activities ( $n=54,16.0 \%)$ compared to men $(n=33,9.8 \%)$. A higher number of men $(n=75,22.3 \%)$ met weekly PA targets through leisure-related activities compared to women $(n=54,16.1 \%)$. A higher proportion of women $(38.4 \%, n=129)$ did not attain WHO recommended levels of weekly PA from leisure PA compared to men $(23.2 \%$, $n=78)$. Overall, a higher proportion of women $(n=111,33.7 \%)$ failed to achieve weekly total PA targets compared to men $(n=80,24.3 \%)$.

\subsection{Multiple Logistic Regressions}

We used multiple logistic regression modeling to assess associations between BE factors and meeting WHO-recommended levels of domain-specific PA (leisure, travel, and total) among men and women. Unadjusted and adjusted odds ratios examining these associations among men and women are presented in Tables 4 and 5 respectively. Land use mix diversity and safety from traffic were the only BE factors that significantly improved travel-related PA among men. The odds of travel PA increased two and a half fold in neighborhoods with greater safety from traffic $(\mathrm{OR}=2.5, \mathrm{Cl}=1.1,5.5)$ and four-fold with greater land-use mix diversity $(\mathrm{OR}=4.0$, 
$1 \mathrm{Cl}=1.7,9.1)$. There was a significant three-fold increase in leisure PA with street connectivity

$2(\mathrm{OR}=2.9, \mathrm{Cl}=1.3,6.6)$. Land use mix diversity significantly lowered the odds of engagement in

3 leisure $\mathrm{PA}$ by $70 \%(\mathrm{OR}=0.3, \mathrm{Cl}=0.1,0.3)$. None of the other $\mathrm{BE}$ factors were significantly

4 associated with leisure PA. Land-use mix diversity $(\mathrm{aOR}=1.4, \mathrm{Cl}=0.5,3.9)$ and safety from

5 traffic $(\mathrm{aOR}=1.1, \mathrm{Cl}=0.5,2.3)$ were the only factors positively related to total $\mathrm{PA}$ in the adjusted

6 models.

Table 5 shows BE predictors of PA among women. Travel PA increased three-fold with greater street connectivity $(\mathrm{aOR}=3.2, \mathrm{Cl}=1.3,8.0)$ and approximately four-fold with increased land-use mix access $(\mathrm{aOR}=3.7, \mathrm{Cl}=1.4,8.2)$. Greater residential density increased the likelihood of travel

$11 \mathrm{PA}$ among women $(\mathrm{OR}=1.7, \mathrm{Cl}=1.1,4.3)$, but this relationship was not statistically significant.

12 The unadjusted odds of travel PA increased with a mix of land-uses $(\mathrm{OR}=1.3, \mathrm{Cl}=0.6,2.6)$ and

13 infrastructure for walking and cycling $(\mathrm{OR}=1.5, \mathrm{Cl}=0.5,3.2)$, but these relationships were not 14 significant and weakened in adjusted models. No significant correlations were found between BE characteristics and leisure PA in women. However, four BE characteristics-land-use mix diversity, infrastructure for walking and cycling, aesthetics and safety from crime-were positively associated with leisure PA among women. The adjusted odds of total PA in women significantly increased with land use mix access $(\mathrm{aOR}=2.4, \mathrm{Cl}=1.1,5.0)$. Although not statistically significant, the adjusted odds of travel PA improved with better street connectivity $(\mathrm{aOR}=1.6, \mathrm{Cl}=0.7,3.6)$.

The unadjusted odds of travel PA in women significantly increased with overall walkability as measured by the aggregate NEWS-India score $(\mathrm{OR}=2.1, \mathrm{Cl}=1.1,4.1)$. Higher aggregate NEWS-India score decreased likelihood of engagement in leisure PA in men $(\mathrm{aOR}=0.6, \mathrm{Cl}=0.3$, 1.3) and women $(\mathrm{aOR}=0.8, \mathrm{Cl}=0.4,1.5)$, but these associations were not significant. Adjusted odds of travel PA were positively associated with aggregate NEWS-India scores in both men $(\mathrm{aOR}=1.4, \mathrm{Cl}=0.6,3.2)$ and women $(\mathrm{aOR}=1.7, \mathrm{Cl}=0.8,3.3)$. 


\section{Discussion}

2 In this study, we examined differences in walkability, transport and PA by gender in the city of

3 Chennai, India. Findings highlight declining trends in PA among women in urban areas and

4 socio-cultural norms that engender differences in PA participation. This study is one of the first

Previous population surveys of risk factors for NCD's in India have found that physical activity levels in rural women were seven times higher than urban women. ${ }^{33-36}$ Obesity rates were highest among women in urban areas and lowest in rural women. ${ }^{32,33}$ Urbanization is linked with a rise in NCD risk factors like physical inactivity, and women are more likely to be inactive as compared to men. ${ }^{37,}{ }^{32}$ Results from this study confirm the higher prevalence of NCD risk factors such as low PA among urban women in Chennai.

Women represent the largest share of public transport users in LMICs, yet they face many barriers that limit their mobility. In our analyses, a higher proportion of women reported achieving recommended PA targets from transport-related activities, walking, and cycling, compared to men. Although walking and bicycling are still common means of transportation in India, accounting for $50 \%$ to $70 \%$ of commuter trips in some urban areas, social prejudice against bicycle use prevails, and car ownership is an indicator of higher social status. ${ }^{38}$ Compared to developed nations, motor vehicle ownership rates are lower in LMICs, especially among women in lower income groups. In these contexts where women have limited travel mode choices, walking, bicycling, and use of public transport are indispensable modes for commuting.$^{39}$ Findings from this study corroborate a multi-country IPEN study including LMICs such as Hong Kong, Mexico, Colombia, and Brazil with low levels of car ownership in the population, thus necessitating the use of active travel modes ${ }^{40}$ However, in the last decade, ownership of private motor vehicles (car and motorcycles) has significantly increased in India, 
1 accounting for about $30 \%$ of the total transport demand. ${ }^{38,41}$ The juxtaposition of different

2 means of transportation (pedestrians, bicyclists, and drivers), weak road regulations and poor

3 law enforcement contribute to India's high numbers of road accidents and traffic fatalities. ${ }^{42}$

4 Under these circumstances, promoting active transportation as a recommendation for the population must go hand-in-hand with policies and interventions for improvement of road safety.

7 Use of public transportation is a known facilitator for PA, yet lack of safety from crime,

8 inconsistent last-mile connectivity, and poor access to transit stops like bus and train stations

9 outweigh women's use of public transport services in India. Commute patterns in India vary

10 distinctly by socioeconomic status-low socioeconomic groups are more likely to use walk,

11 bicycle or public transportation in India compared to high-income groups. ${ }^{18}$ In contrast, women in middle-to-high-income groups prefer to use private motorized transport, mainly cars, scooters, and motorcycles, even for short distances. Commonly cited deterrents to walking among women include lack of infrastructure for walking, traffic congestion and poor street connectivity, which is consistent with the findings from this study. Similar to other studies from highly urbanized and rapidly urbanizing LMICs, we found positive correlations of total PA with land use mix-access and street connectivity. ${ }^{43}$

In contrast to transportation PA, the proportion of women engaging in leisure-time PA found in this study was lower, thus emphasizing the need to implement comprehensive methods that balance the promotion of PA through various domains. Low engagement in leisure-time PA among women may be linked to prevailing cultural and social norms that constrain women's' mobility and hinder their participation in PA. For example, cycling is perceived as more appropriate for younger men, but an improper and unacceptable activity for women to engage in as per societal traditions. ${ }^{18}$ Some women do not prefer using bicycles due to the inconvenience caused by the local attire (sari). ${ }^{18}$

Other barriers include negative perceptions of active travel, low self-esteem due to poor body image, time constraints due to household and caregiving responsibilities, low motivation, and 
1 disinclination toward any types of PA. ${ }^{18}$ Women from low-income groups may not have the

2 financial means to afford services such as housecleaning or childcare to relieve them for 3 leisure-PA participation. ${ }^{44,45}$ They may also be constrained in accessing fee-based leisure-PA opportunities (e.g., gyms, recreation centers, sports clubs) or unable to visit a safe outdoor neighborhood space such as a park or playground, further limiting their ability to engage in leisure-time PA. ${ }^{45}$ Due to gendered social roles, women may be active in other domains (e.g., domestic or occupational) rather than during leisure, which should be explored in future research.

\subsection{Strengths and Limitations}

In this study, gender was defined as socio-cultural constructs, norms and expectations based on binary biological characteristics, male and female. Therefore, other gender(s) and their access to mobility/transport were not explored. The small sample size from a single city in India and cross-sectional nature of the study design limits the ability to generalize findings and make any inferences of causality. This study relied on participants' own reporting of their levels of PA and perceptions of the BE. Self-reported data has its limitations as individuals may be biased when reporting their own experiences. For example, participants are likely to respond in a way that is socially desired or acceptable, which may result inaccurate estimations of PA levels. Physically active individuals may observe and report more opportunities for PA and supportive neighborhood infrastructure than those who are less active.

The validity and reliability of instruments that were used for data collection must be considered while interpreting the results of this study. The IPAQ is a standardized, internationally validated tool for measuring PA, however the domains used in the current study may not have fully vigorous in others. In India, PA associated with transportation is a regular occurrence in urban areas where an individual's daily routine involves travel to work. On the contrary, leisure-time 
1 PA may not be commonplace in the participants of this study given the socio-cultural setting of

2 the population.

4 This study did not measure domestic and occupational levels of PA, which is another limitation

5 of this study. Household chores requiring movement (e.g., cleaning, vacuuming, gardening) are

6 known to be a key source of energy expenditure among women. ${ }^{46}$ This is especially important

7 in India as domestic duties may often be divided along gender lines, and many wealthier

8 households may employ women as domestic helpers. This study did not measure rural PA

9 patterns, where women may undertake vigorous farming work for long hours involving

10 ploughing, tilling fields and shepherding livestock. Performing these vigorous domestic and

11 occupational activities can add up to reach the recommended PA levels among women. ${ }^{47}$

13 Despite these limitations, this study has notable strengths. As part of IPEN, rigorous methods

14 and measures were used. ${ }^{48}$ Given the limited research on this topic, our findings highlight 15 gender-specific associations between PA and BE in India, that are noticeably different from 16 patterns observed in developed countries. Our findings also call attention to the need to 17 integrate gender-responsive policies across urban planning, transport, and public health sectors in LMICs.

\section{Conclusion}

21 A majority of the world's population now lives in cities with women comprising half of the population, therefore research exploring the intersection of health disparities, gender roles and cultural issues in urban planning and transportation is paramount. ${ }^{49}$ Public health practitioners must address gender-specific barriers to increase PA among women. Health promotion strategies and interventions should be designed to suit the predominant socio-cultural norms in the community, improve self-efficacy, and enhance social support among women through context-specific group exercise programs, peer support and guidance that emphasizes the importance of PA. ${ }^{18}$ 
1 India's efforts to address gender inequalities in PA will be critical to attaining the global action

2 plan targets set by the World Health Organisation. In the past decade, several programs and

3 policies have been launched across India to promote sustainable, inclusive, and healthy cities.

4 The Government of India's on-going '100 Smart Cities Mission' aspires to renew and retrofit 100

5 cities across the country with walkable neighborhoods, improved access to parks, green

6 spaces, and public transit infrastructure. ${ }^{50}$ In LMICs like India, aspects of gender equity in

7 transportation planning are closely linked with citywide indicators of liveability, sustainability,

8 and resilience and must be integrated together with smart city planning features. India's

9 strategy for the prevention and control of NCDs needs to respond to the challenges of

10 urbanization and warrants a closer look into the creation of safe, gender-sensitive and culturally

11 acceptable strategies to encourage women's participation in PA. 


\section{Abbreviations}

NCD Non-Communicable Disease

LMICs Low- and Middle-Income Countries

PA Physical Activity

$\mathrm{BE}$

Built Environment

IPEN International Physical activity and the Environment Network

NEWS Neighborhood Environment Walkability Scale

IPAQ-LF International Physical Activity Questionnaire-Long Form

\section{Acknowledgements}

We thank Dr. James F. Sallis (University of California, San Diego) and members of the IPEN Coordinating Center for their assistance with methods and study protocols. We also acknowledge the team of research assistants that assisted with translation and data collection in India: S. Soundhariya, Priyadarshini Chidambaram, S. Gayathri, R. Dhivya, Gomathi Srinivasan, V. Sridevi, and V. Shanthi. 


\section{References}

1. Lachat C, Otchere S, Roberfroid D, Abdulai A, Seret FMA, Milesevic J, et al. Diet and Physical Activity for the Prevention of Noncommunicable Diseases in Low- and Middle-Income Countries: A Systematic Policy Review. PLOS Medicine 2013;10(6):e1001465.

2. Pratt M, Norris J, Lobelo F, Roux L, Wang G. The cost of physical inactivity: moving into the 21st century. Br J Sports Med 2014;48:171-173.

3. Boutayeb A, Boutayeb S. The burden of non communicable diseases in developing countries. International Journal for Equity in Health 2005;4(1):2.

4. Ranasinghe PD, Pokhrel S, Anokye NK. The economics of physical activity in lowincome and middle-income countries: protocol for a systematic review. BMJ open 2019;9(1):e022686-e022686.

5. Shrivastava U, Misra A, Mohan V, Unnikrishnan R, Bachani D. Obesity, Diabetes and Cardiovascular Diseases in India: Public Health Challenges. Curr Diabetes Rev 2017;13(1):6580 .

6. Upadhyay RP. An overview of the burden of non-communicable diseases in India. Iranian journal of public health 2012;41(3):1-8.

7. Agrawal R, Chaturvedi M, Singh S, Gupta SC. An epidemiological study of dietary and exercise habits as correlates of hypertension in persons aged 45 years and above in Agra District. Indian J Comm Health 2012;24(2):91 - 96.

8. Bloom DE, Cafiero-Fonseca ET, Candeias V, Adashi E, Bloom L, Gurfein L, et al. Economics of Non-Communicable Diseases in India: The Costs and Returns on Investment of Interventions to Promote Healthy Living and Prevent, Treat, and Manage NCDs. Geneva, Switzerland: World Economic Forum, Harvard School of Public Health; 2014.

9. Editorial. Time to tackle the physical activity gender gap. The Lancet Public Health 2019;4(8):e360.

10. Chopra SM, Misra A, Gulati S, Gupta R. Overweight, obesity and related noncommunicable diseases in Asian Indian girls and women. Eur J Clin Nutr 2013;67(7):688-96.

11. World Health Organization. 2010. Switzerland: World Health Organization; Global recommendations on physical activity for health.

12. US Department of Health and Human Services. Physical Activity Guidelines for Americans; 2018.

13. UK Department of Health and Social Care. UK Physical Activity Guidelines; 2011. 14. Morgan SP, Stash S, Smith HL, Mason KO. Muslim and Non-Muslim Differences in Female Autonomy and Fertility: Evidence from Four Asian Countries. Population and Development Review 2002;28(3):515-537.

15. Moreno JP, Johnston CA. Barriers to Physical Activity in Women. American Journal of Lifestyle Medicine 2014;8(3):164-166.

16. Adlakha D, Hipp JA, Brownson RC. Adaptation and Evaluation of the Neighborhood Environment Walkability Scale in India (NEWS-India). International Journal of Environmental Research and Public Health 2016;13(4):401.

17. Babakus WS, Thompson JL. Physical activity among South Asian women: a systematic, mixed-methods review. International Journal of Behavioral Nutrition and Physical Activity 2012;9(1):150.

18. Mathews E, Lakshmi JK, Ravindran TK, Pratt M, Thankappan KR. Perceptions of barriers and facilitators in physical activity participation among women in Thiruvananthapuram City, India. Glob Health Promot 2016;23(4):27-36.

19. Lei L, Desai S, Vanneman R. The Impact of Transportation Infrastructure on Women's Employment in India. Feminist Economics 2019;25(4):94-125.

20. Ministry of Home Affairs. Census of India 2011. New Delhi, India: Government of India; 2013.

21. Elshahat S, O'Rorke M, Adlakha D. Built environment correlates of physical activity in low- and middle-income countries: A systematic review. PloS one 2020;15(3):e0230454e0230454.

22. consortium CW. Gender equality and mobility: mind the gap!: CIVITAS; 2020.

23. Kerr J, Sallis JF, Owen N, De Bourdeaudhuij I, Cerin E, Sugiyama T, et al. Advancing science and policy through a coordinated international study of physical activity and built environments: IPEN adult methods. J Phys Act Health 2013;10(4):581-601. 
24. Adams MA, Frank LD, Schipperijn J, Smith G, Chapman J, Christiansen LB, et al. International variation in neighborhood walkability, transit, and recreation environments using geographic information systems: the IPEN adult study. International Journal of Health Geographics 2014;13(1):43.

25. Oyeyemi AL, Sallis JF, Deforche B, Oyeyemi AY, De Bourdeaudhuij I, Van Dyck D. Evaluation of the neighborhood environment walkability scale in Nigeria. International Journal of Health Geographics 2013;12:16.

26. Adlakha D, Hipp JA, Brownson RC. Neighborhood-based differences in walkability, physical activity, and weight status in India. Journal of Transport \& Health 2016;3(4):485-499. 27. Cerin E, Conway TL, Cain KL, Kerr J, De Bourdeaudhuij I, Owen N, et al. Sharing good NEWS across the world: developing comparable scores across 12 countries for the Neighborhood Environment Walkability Scale (NEWS). BMC Public Health 2013;13:309. 28. Parra DC, Hoehner CM, Hallal PC, Ribeiro IC, Reis RS, Brownson RC, et al. Perceived environmental correlates of physical activity for leisure and transportation in Curitiba, Brazil. Prev Med 2011;52(3-4):234-8.

29. IPAQ. International physical activity questionnaires. IPAQ-long: last 7 days selfadministered format. In: International physical activity questionnaires; 2002.

30. Hallal PC, Victora CG. Reliability and validity of the International Physical Activity Questionnaire (IPAQ). Med Sci Sports Exerc 2004;36(3):556.

31. IBM Corp. IBM SPSS Statistics for Windows Version 25.0. In. Armonk, NY: IBM Corp.; 2017.

32. Sengupta A, Angeli F, Syamala TS, Dagnelie PC, van Schayck CP. Overweight and obesity prevalence among Indian women by place of residence and socio-economic status: Contrasting patterns from 'underweight states' and 'overweight states' of India. Soc Sci Med 2015;138:161-9.

33. Yadav K, Krishnan A. Changing patterns of diet, physical activity and obesity among urban, rural and slum populations in north India. Obes Rev 2008;9(5):400-8.

34. Pradeepa R, Anjana RM, Joshi SR, Bhansali A, Deepa M, Joshi PP, et al. Prevalence of generalized \& abdominal obesity in urban \& rural India--the ICMR-INDIAB Study (Phase-I) [ICMR- NDIAB-3]. Indian J Med Res 2015;142(2):139-50.

35. Meshram, II, Vishnu Vardhana Rao M, Sudershan Rao V, Laxmaiah A, Polasa K. Regional variation in the prevalence of overweight/obesity, hypertension and diabetes and their correlates among the adult rural population in India. Br J Nutr 2016;115(7):1265-72.

36. Garg C, Khan SA, Ansari SH, Garg M. Prevalence of obesity in Indian women. Obes Rev 2010;11(2):105-8.

37. Ogden CL, Fryar CD, Hales CM, Carroll MD, Aoki Y, Freedman DS. Differences in Obesity Prevalence by Demographics and Urbanization in US Children and Adolescents, 20132016Obesity Prevalence by Demographics and Urbanization in US Youth, 2013-2016Obesity Prevalence by Demographics and Urbanization in US Youth, 2013-2016. JAMA 2018;319(23):2410-2418.

38. Ministry of Road Transport \& Highways. Road Transport Yearbook 2007 - 2009 (Volume- I). New Delhi: Transport Research Wing, Government of India; 2011.

39. Shoham DA, Dugas LR, Bovet P, Forrester TE, Lambert EV, Plange-Rhule J, et al. Association of car ownership and physical activity across the spectrum of human development: Modeling the Epidemiologic Transition Study (METS). BMC public health 2015;15:173-173.

40. Sallis JF, Cerin E, Conway TL, Adams MA, Frank LD, Pratt M, et al. Physical activity in relation to urban environments in 14 cities worldwide: a cross-sectional study. Lancet 2016;387(10034):2207-17.

41. Ministry of Road Transport and Highways. Basic Road Statistics of India. In: Ministry of Road Transport and Highways; 2010-2011.

42. World Health Organization. Global status report on road safety: time for action Geneva, Switzerland; 2009.

43. Gomez LF, Sarmiento R, Ordonez MF, Pardo CF, de Sa TH, Mallarino CH, et al. Urban environment interventions linked to the promotion of physical activity: a mixed methods study applied to the urban context of Latin America. Soc Sci Med 2015;131:18-30.

44. Adlakha D, Hipp JA, Brownson RC, A AE, C KL, Raghavan R. "Can we walk?" Environmental supports for physical activity in India. Prev Med 2017;103s:S81-s89. 
45. Gómez LF, Mateus JC, Cabrera G. Leisure-time physical activity among women in a neighbourhood in Bogotá, Colombia: prevalence and socio-demographic correlates. Cadernos de Saúde Pública 2004;20:1103-1109.

46. Phongsavan P, Merom D, Marshall A, Bauman A. Estimating physical activity level: the role of domestic activities. Journal of Epidemiology and Community Health 2004;58(6):466-467. 47. Archer E, Shook RP, Thomas DM, Church TS, Katzmarzyk PT, Hébert JR, et al. 45Year Trends in Women's Use of Time and Household Management Energy Expenditure. PLOS One 2013.

48. Kerr J, Emond JA, Badland H, Reis R, Sarmiento O, Carlson J, et al. Perceived Neighborhood Environmental Attributes Associated with Walking and Cycling for Transport among Adult Residents of 17 Cities in 12 Countries: The IPEN Study. Environ Health Perspect 2016.

49. United Nations. The World's Cities in 2018: United Nations, Department of Economic and Social Affairs, Population Division; 2018.

50. Government of India. Smart Cities Mission. New Delhi, India; 2015. 